\title{
Nitric oxide/cyclic GMP pathway mediates the endothelin-1-upregulation of adiponectin expression in rat cardiomyocytes
}

\author{
BINGYAN GUO, YONGJUN LI, XIN JIN, SUYUN LIU and CHENGLONG MIAO \\ Department of Cardiovascular Medicine, The Second Hospital of Hebei \\ Medical University, Shijiazhuang, Hebei 050017, P.R. China
}

Received June 14, 2016; Accepted September 21, 2016

DOI: $10.3892 /$ br.2017.953

\begin{abstract}
Endothelin-1 (ET-1) serves an important role in the development of cardiac dysfunction and heart failure. ET-1 and angiotensin II (AngII) comprise a mutually reciprocal signalling network in the myocardium and serve similar or additive roles in the development of heart failure. Our previous study previously demonstrated that AngII upregulated the expression of APN in cardiomyocytes through an AngII receptor type 2/nitric oxide (NO)/cyclic GMP(cGMP)-dependent pathway. The purpose of the present study was to determine the effects of ET-1 and the additive effects of ET-1 and AngII on the gene expression and secretion of APN, and the underlying mechanisms involved. ELISA was used to determine the secretion of adiponectin (APN) and reverse transcription-quantitative polymerase chain reaction was used to evaluate the gene expression of APN. ET-1 induced APN secretion in a time- and dose-dependent manner, and induced APN secretion with AngII simultaneously, as determined via APN mRNA analyses. ETA and ETB receptors were also involved. The use of a NO synthase inhibitor and an analogue of cGMP antagonist resulted in a diminished ET-1- and/or AngII-mediated APN induction in cardiomyocytes. These results suggested that ET-1, as well as AngII, upregulated the gene expression and secretion of APN via the common NO/cGMP-mediated mechanism.
\end{abstract}

\section{Introduction}

A series of complex and severe neurohormonal abnormalities, including increased levels of cardiac and circulating

Correspondence to: Professor Yongjun Li, Department of Cardiovascular Medicine, The Second Hospital of Hebei Medical University, 215 Peace West Road, Shijiazhuang, Hebei 050017, P.R. China

E-mail: gbyan2008bs@163.com

Key words: angiotensin II, adiponectin, endothelin-1, myocardium, nitric oxide, cGMP endothelin-1 (ET-1) and angiotensin II (AngII), have been linked to the development of cardiac dysfunction and heart failure. It has been demonstrated that ET-1 and AngII comprise a mutually reciprocal signalling network and exhibit certain reciprocal effects on the myocardium. In previous studies using cultured neonatal rat ventricular myocytes (NRVMs), endogenous ET-1 mediates AngII-induced hypertrophy (1). The exogenous administration of ET-1 also induced hypertrophic responses in cardiomyocytes (2). As the predominant receptors on myocytes, AngII receptor type-1 $\left(\mathrm{AT}_{1} \mathrm{R}\right)$ and ET type A receptors $\left(E_{A} R\right)$, as well as AngII receptor type-2 $\left(A_{2} R\right)$ and ET type $B$ receptors $\left(E_{B} R\right)$, share several common subcellular signalling pathways (3). As a result of these local positive feedback loops and signal redundancy, the roles of these receptors in chronic processes, including in vivo hypertrophy and cardiac failure, were synergistic or additive.

Adiponectin (APN), a circulating cytokine, has been shown to be produced by adipocytes, skeletal myocytes, endothelial cells, as well as cardiomyocytes, and serves an important role in glucose and lipid metabolism (4,5). Numerous previous reports have demonstrated increased APN levels in the peripheral circulation in patients with chronic heart failure (CHF); high plasma APN levels are significant prognostic indicators in these patients (6-8). Several previous reports have postulated roles for ET-1 in the regulation of APN production in adipocytes and cardiomyocytes. In cultured human cardiomyocytes, ET-1 has been reported to cause a significant increase in myocyte size and the protein expression of APN (9). However, the underlying mechanisms remain unknown. It is known that ET-1 can bind to $\mathrm{ET}_{\mathrm{B}} \mathrm{R}$ to stimulate nitric oxide (NO) production, resulting in an increased formation of the second messenger, cyclic GMP(cGMP)-dependent protein kinase. Our previous study demonstrated a concentration-dependent increase in the mRNA expression of APN when NRVMs were exposed to sodium nitroprusside (SNP), a NO donor, as well as a cGMP agonist, 8-Br-cGMP. AngII increased the production of APN in NRVMs through NO/cGMP activation. Cardiomyocyte-produced APN may be the major source of the observed upregulation in circulating APN levels in CHF (10). Whether ET-1 and AngII serve alternative, additive, or synergistic roles in APN activation via the identical NO/cGMP signalling pathway in NRVMs remains to be elucidated. 
The present study sought to address the following: i) Whether ET-1 upregulates APN gene expression and secretion in cardiomyocytes; ii) whether ET-1 and AngII serve additive roles in APN gene expression and secretion; iii) to determine the molecular mechanism underlying the activation of APN in response to ET-1 and AngII. The present results demonstrated that ET-1-induced upregulation of the gene expression and secretion of APN potentially occurs via the ETA and ETB receptors. ET-1 and AngII were additive in the activation of APN. The use of an NO synthase inhibitor (Nx-nitro-L-arginine methyl ester hydrochloride) and an analogue of the cGMP antagonist (Rp-8-Br-CGMP-S) resulted in the diminished additive roles of ET-1 and AngII on APN induction in cardiomyocytes.

\section{Materials and methods}

Materials. Dulbecco's modified Eagle's medium (DMEM), penicillin, and streptomycin were obtained from Life Technologies (Thermo Fisher Scientific, Inc., Waltham, MA, USA). Telmisartan, PD123319, BQ-610 and BQ-788 were purchased from Phoenix Pharmaceutical, Inc. (Belmont, CA, USA). ET-1, AngII, actinomycin D, bovine serum albumin and all other chemicals were obtained from Sigma-Aldrich (St. Louis, MO, USA).

Primary culture of NRVMs. NRVMs were prepared from ventricles of 1-3-day-old Sprague-Dawley rats, as previously described (10). Briefly, following digestion in phosphate-buffered saline containing $0.1 \%$ trypsin and $0.04 \%$ type II collagenase, the cells were centrifuged at $320 \mathrm{x}$ g at $37^{\circ} \mathrm{C}$ for $5 \mathrm{~min}$ and suspended in DMEM containing $15 \%$ fetal calf serum (FCS). The cells were pre-seeded and cultured for $2 \mathrm{~h}$ to eliminate non-myocardial cells. Non-attached cells were seeded at $1 \times 10^{6}$ cells $/ \mathrm{cm}^{2}$ in the same medium as above. Following incubation, the cells were washed and the medium was replaced with DMEM containing $0.5 \%$ FCS for $24 \mathrm{~h}$ prior to each experiment.

ELISA. Following various treatments, the medium was collected and stored at $-80^{\circ} \mathrm{C}$ for future use. APN contents in the samples from the same set of experiments were determined using an ELISA (R\&D Systems, Inc., Minneapolis, MN, USA), according to the manufacturer's protocol.

Reverse transcription-quantitative polymerase chain recation $(R T-q P C R)$. The total RNA was extracted from the cells using TRIzol reagent (Invitrogen; Thermo Fisher Scientific, Inc.), according to the manufacturer's protocol. cDNA was synthesised using a TaqMan RT kit (Applied Biosystems; Thermo Fisher Scientific, Inc.). The qPCR was performed using the following reaction: $1 \mathrm{X}$ gene master mix and $1 \mathrm{X}$ APN primer and probe mix (cat. no. Hs00152932 m1; Applied Biosystems; Thermo Fisher Scientific, Inc.) in $25 \mu 1$ reactions in a 96-well plate. The reactions were performed in duplicate for each sample. The plate was then placed in an ABI Prism 7500 Sequence detection system (Applied Biosystems; Thermo Fisher Scientific, Inc.). PCR cycles were performed 40 times using a three-step cycle procedure (denaturation at $94^{\circ} \mathrm{C}$ for $30 \mathrm{sec}$, annealing at $58^{\circ} \mathrm{C}$ for $30 \mathrm{sec}$ and extension at $72^{\circ} \mathrm{C}$ for
$30 \mathrm{sec}$ ), following the initial stage at $96^{\circ} \mathrm{C}$ for $4 \mathrm{~min}$. Glyceraldehyde 3-phosphate dehydrogenase (GAPDH) RNA (Applied Biosystems; Thermo Fisher Scientific, Inc.) was used as the endogenous control. For the comparison of APN with GAPDH RNA, the cycle threshold value was analysed (Cq) using the $2^{-\Delta \Delta C q}$ method (11). The data were subsequently reported as the fold-change compared with the control. The primer sequence for APN was as follows: Sense, 5'-GCCGTT CTCTTCACCTACGA-3' and antisense, 5'-TGGTCTCCC ACCTCCAGAT-3'.

Statistical analysis. The data are presented as the mean \pm standard error of the mean. Group mean values were compared using a one-way analysis of variance, followed by Tukey's multiple comparison test, where appropriate. Statistics were performed using GraphPad/Prism 5 software (GraphPad Software, Inc., La Jolla, CA, USA). P $<0.05$ was considered to indicate a statistically significant difference.

\section{Results}

ET-1 upregulates APN secretion and exerts additive effects with AngII on APN secretion in NRVMs. To determine effect of ET-1 and AngII on APN secretion, NRVMs were treated with ET-1 for $24 \mathrm{~h}$ and the released APN in the medium was measured. As shown in Fig. 1A, ET-1 upregulated the secretion of APN, reaching a maximum of $10 \mathrm{nM}$ from 1.0-6.52 \pm 0.52 -fold induction $(\mathrm{P}<0.01 ; \mathrm{n}=4)$. When compared with ET-1 alone, the combination of ET-1 and AngII simultaneously induced higher levels of APN (between $2.31 \pm 0.23$ and $9.98 \pm 0.77$-fold induction; $\mathrm{P}<0.01 ; \mathrm{n}=4$ ). Similarly, an additive effect of between $10 \mathrm{nM}$ AngII and varying concentrations of ET-1 on the release of APN was also observed (Fig. 1B). Since the maximal effect was observed at $10 \mathrm{nM}$ ET-1 and $10 \mathrm{nM}$ AngII, these concentrations were used throughout the present study. Additionally, the time-dependent effects of ET-1, and a combination of ET-1 and AngII also demonstrated the additive effect on APN secretion (Fig. 1C).

ET-1 upregulates APN gene expression and exerts additive effects with AngII on the gene expression and secretion of APN in NRVMs. Since the secretory process of APN in adipocytes is likely to be subject to its gene expression, the enhancing effect of ET-1 on APN secretion in NRVMs is most likely also due to augmented gene expression. To assess this, a transcription inhibitor (actinomycin D) was used. As shown in Fig. 2A, in the presence of actinomycin D, the enhancing effect of ET-1, AngII and ET-1 plus AngII was inhibited by 95, 92 and 82\%, respectively, suggesting that the primary target site of regulation by ET-1 and AngII is the APN gene.

To further determine whether the gene expression of APN is similarly enhanced by ET-1 and AngII, the cells were treated with ET-1, AngII and a combination of both, and were subsequently evaluated using RT-qPCR. As shown in Fig. 2B, the mRNA expression of APN was augmented by ET-1 and AngII in an additive manner.

$E T_{A} R$ and $E T_{B} R$ as well as $A T_{2} R$ were all involved in the effect of ET-1 and AngII on APN gene expression. Although it was previously demonstrated that AngII upregulated the expression 
A

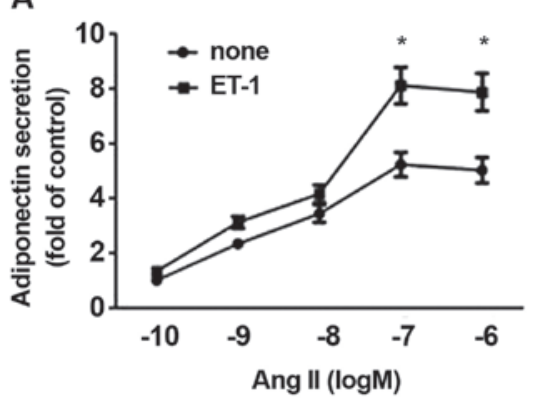

C

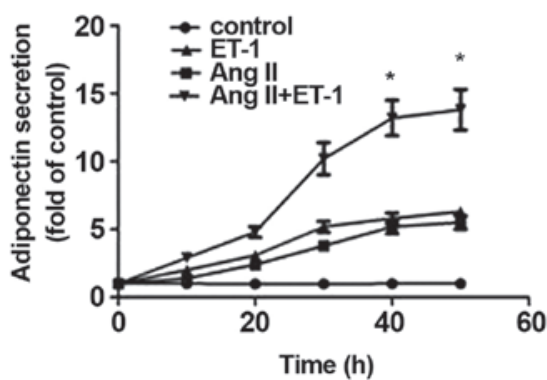

$\mathbf{B}$

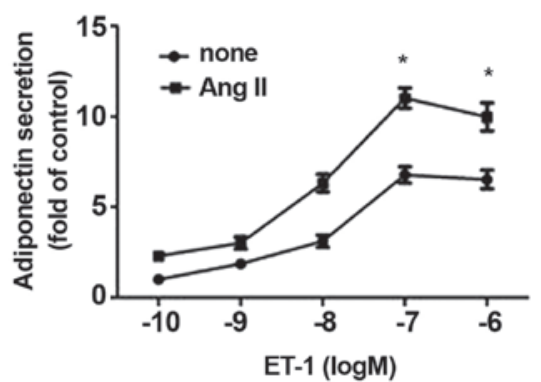

Figure 1. Combined effects of ET-1 and AngII on APN release from NRVMs. NRVMs were treated with (A) varying concentrations of AngII alone or in combination with $10 \mathrm{nM}$ ET-1, or with (B) varying concentrations of ET-1 alone or in combination with $10 \mathrm{nM}$ AngII for 24 h. The medium was assessed to determine APN content using ELISA. (C) Time-dependent effect of ET-1, AngII or a combination of each on the release of APN. NRVMs were treated without (control) or with $10 \mathrm{nM}$ ET-1, AngII or both for 8, 16, 24 and $48 \mathrm{~h}$ and APN release was assessed. The data are presented as the mean \pm standard error of the mean of four separate experiments, ${ }^{*} \mathrm{P}<0.05$ compared with the corresponding control. ET-1, endothelin-1; AngII, angiotensin II; APN, adiponectin; NRVMs, neonatal rat ventricular myocytes.

of APN in NRVMs via the $\mathrm{AT}_{2} \mathrm{R}$ and that ET-1 downregulated APN gene expression via the $\mathrm{ET}_{\mathrm{A}} \mathrm{R}$ activation in 3T3-L1 adipocytes, the additive effects of ET-1 and AngII on APN secretion may not be mediated by the same receptor. Since the additive effect of ET-1 and AngII is predominantly attributable to their effects on APN gene expression, the present study employed the gene assay to assess which ET and AngII receptor was involved; this was performed using BQ610 and BQ788, which are selective antagonists for ETA and ETB receptors, as well as telmisartan and PD123319, which are selective antagonists for $\mathrm{AT}_{1}$ and $\mathrm{AT}_{2} \mathrm{Rs}$, respectively. As shown in Fig. 3, AngII-stimulated APN gene expression was inhibited by PD123319 $(\mathrm{P}<0.01, \mathrm{n}=4)$ and telmisartan had a non-significant effect. ET-1-stimulated gene expression of APN was inhibited by BQ610 $(\mathrm{P}<0.01, \mathrm{n}=4)$ and BQ788 $(\mathrm{P}<0.01, \mathrm{n}=4)$. Regarding the gene expression in response to a combination of ET-1 and AngII, BQ610, BQ788 and PD123319, a significantly reduced response was observed. The $\mathrm{AT}_{1}$ receptor was not involved. Using the difference between the increase in gene expression in response to ET-1 plus AngII [(ET-1 + AngII)-(control)] and the sum of the increases caused by ET-1 [(ET-1)-(control)] and AngII [(AngII)-(control)] alone as an index to estimate the additive effect, it was revealed that BQ610 and BQ788, as well as PD123319, inhibited the additive effect by $67(\mathrm{P}<0.01, \mathrm{n}=4), 91$ $(\mathrm{P}<0.01, \mathrm{n}=4)$ and $80 \%(\mathrm{P}<0.01, \mathrm{n}=4)$, respectively. Therefore, it appears that the additive effect of ET-1 and AngII on APN gene expression was mediated by ETA and ETB, as well as $\mathrm{AT}_{2} \mathrm{Rs}$. The ETB receptor may serve a major role.

ET-1 and AngII upregulate the gene expression of APN via the activation of NO/cGMP. To test the hypothesis that the
NO/cGMP pathway serves a role in the additive effects of ET-1 and AngII on APN gene expression, NRVMs were pre-incubated for $1 \mathrm{~h}$ with either L-NAME $\left(10^{-3} \mathrm{M}\right)$ or Rp-8-Br-cGMP-S $\left(10^{-5} \mathrm{M}\right)$, and were then co-incubated with the same inhibitor, as well as $10^{-7} \mathrm{M}$ AngII and/or ET-1 for a further $24 \mathrm{~h}$. As shown in Fig. 4, L-NAME or Rp-8-Br-cGMP-S alone had no effect on the basal mRNA expression of APN; both AngII and ET-1-stimulated APN gene expression was inhibited by L-NAME and Rp-8-Br-cGMP-S, respectively $(\mathrm{P}<0.01, \mathrm{n}=4)$. Regarding the gene expression in response to a combination of ET-1 and AngII, both L-NAME and Rp-8-Br-cGMP-S significantly reduced the response. Using the difference between the increase in gene expression in response to ET-1 plus AngII $[($ ET-1 + AngII)-(control) $]$ and the sum of the increases caused by ET-1 [(ET-1)-(control)] and AngII [(AngII)-(control)] alone as an index to estimate the additive effect, it was calculated that L-NAME and Rp-8-Br-cGMP-S inhibited the additive effect by $87(\mathrm{P}<0.01, \mathrm{n}=4)$ and $91 \%(\mathrm{P}<0.01, \mathrm{n}=4)$, respectively.

\section{Discussion}

The major finding of the present study was that ET-1 upregulated the gene expression and secretion of APN in NRVMs, and the NO/cGMP/PKG signalling pathways mediated this upregulation. ET-1 and AngII caused and additive effect on APN activation.

The APN secretion data in the present study are compatible with those in a previous report in 3T3-L1 adipocytes (12). The authors reported that treatment with ET-1 for $4 \mathrm{~h}$ induced the significant stimulation of APN secretion; however, this effect was lost after $8 \mathrm{~h}$ of treatment. In another previous 

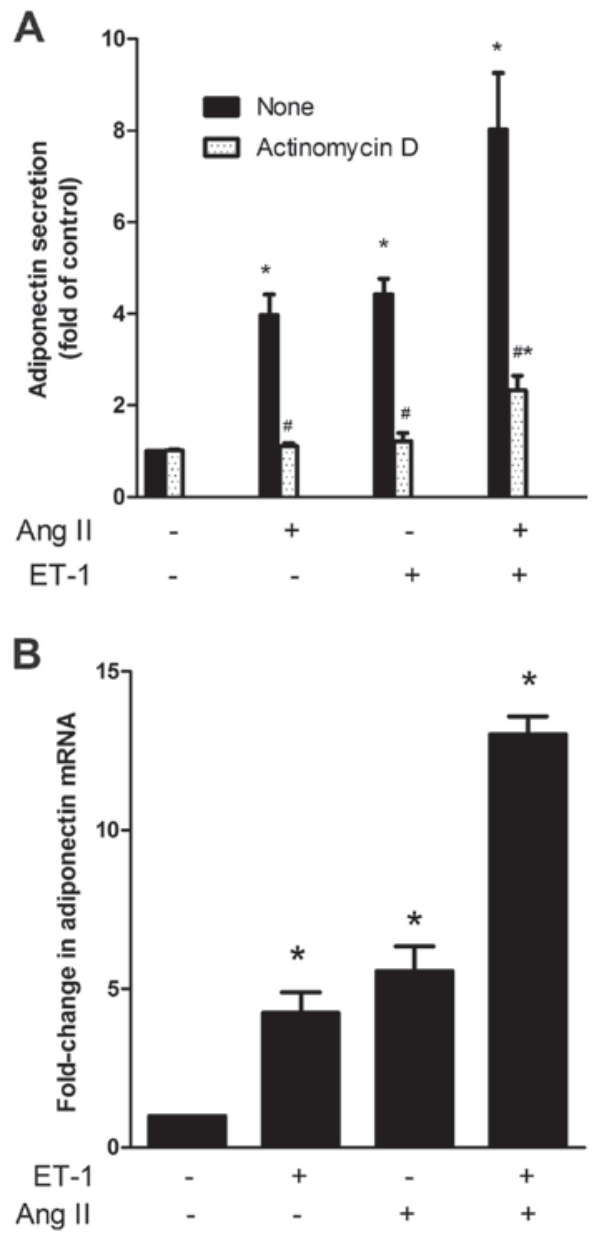

Figure 2. (A) Effect of actinomycin D on APN release induced by ET-1, AngII and a combination of both. NRVMs were treated without or with 5 $\mathrm{mg} / \mathrm{ml}$ actinomycin $\mathrm{D}$ for $30 \mathrm{~min}$ prior to the addition of the vehicle (control), $10 \mathrm{nM}$ ET-1, $10 \mathrm{nM}$ AngII or both for a further $24 \mathrm{~h}$. The medium was measured to determine APN content using ELISA. The data are presented as the mean \pm standard error of the mean ( $\mathrm{P}<0.05$ compared with the corresponding control, ${ }^{,} \mathrm{P}<0.05$ compared with the corresponding untreated group). (B) The effect of ET-1, AngII and a combination of both on the mRNA expression of APN in NRVMs. Following treatment with vehicle (control), ET-1, AngII or both for $24 \mathrm{~h}$, the total RNA was extracted and estimated for APN mRNA expression by reverse transcription-quantitative polymerase chain reaction. The data are presented as the mean \pm standard error of the mean $\left({ }^{*} \mathrm{P}<0.05\right.$ compared with the control). ET-1, endothelin-1; AngII, angiotensin II; APN, adiponectin; NRVMs, neonatal rat ventricular myocytes.

study in 3T3-L1 adipocytes (13), ET-1 exposure for $24 \mathrm{~h}$ led to lower levels of APN secretion compared with the relative control. This different secretion response between NRVMs and 3T3-L1 adipocytes may be due to the different cell type.

Recently, Yin et al (14) suggested that plasma ET-1 and APN increased with the severity of heart failure. APN protein is upregulated in cardiac tissue and is correlated with increased serum concentrations of ET-1, the incubation of HCM with ET-1 significantly increased the protein expression of APN in a time- and dose-dependent manner. However, the possible mechanism responsible for the action of ET-1 was not discussed. The present study revealed that ET-1, acting via $E_{A} R$ and $E_{B} R$, upregulated $A P N$ gene expression. Selective antagonists of $\mathrm{ET}_{\mathrm{A}} \mathrm{R}, \mathrm{ET}_{\mathrm{B}} \mathrm{R}, \mathrm{BQ} 610$ and $\mathrm{BQ788}$ decreased the mRNA expression of APN via inactivation of the NO/cGMP/PKG pathway, since its effect was prevented

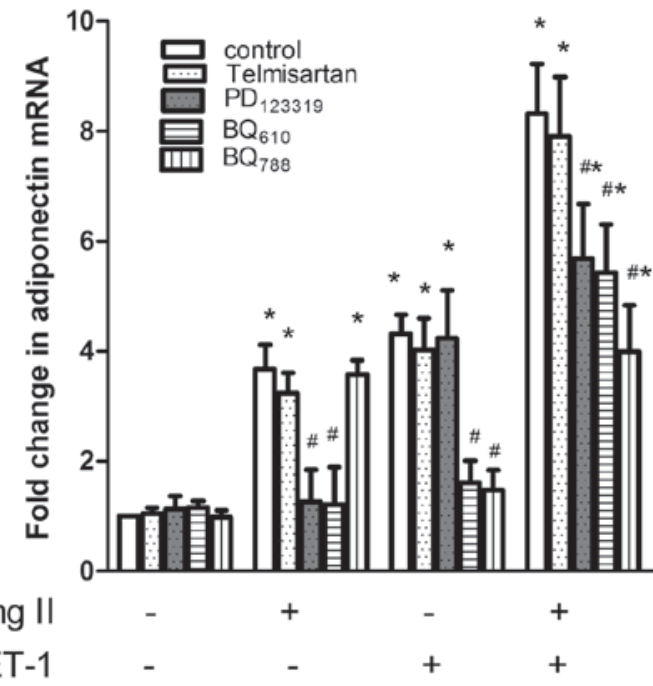

Figure 3. Effect of ET, AngII receptor antagonists on adiponectin mRNA in response to ET-1, AngII and a combination of both drugs. NRVMs were pre-treated with $0,10 \mathrm{mM}$ BQ610, $10 \mathrm{mM}$ BQ788, $10 \mathrm{mM}$ telmisatan or $10 \mathrm{mM}$ PD123319 for $1 \mathrm{~h}$ and the effect of vehicle (control), ET-1, AngII and a combination of both on APN mRNA expression was assessed. The data are presented as the mean \pm standard error of the mean $\left({ }^{*} \mathrm{P}<0.05\right.$ compared with the corresponding control, ${ }^{\text {}} \mathrm{P}<0.05$ compared with the corresponding untreated group). ET-1, endothelin-1; AngII, angiotensin II; APN, adiponectin; NRVMs, neonatal rat ventricular myocytes.

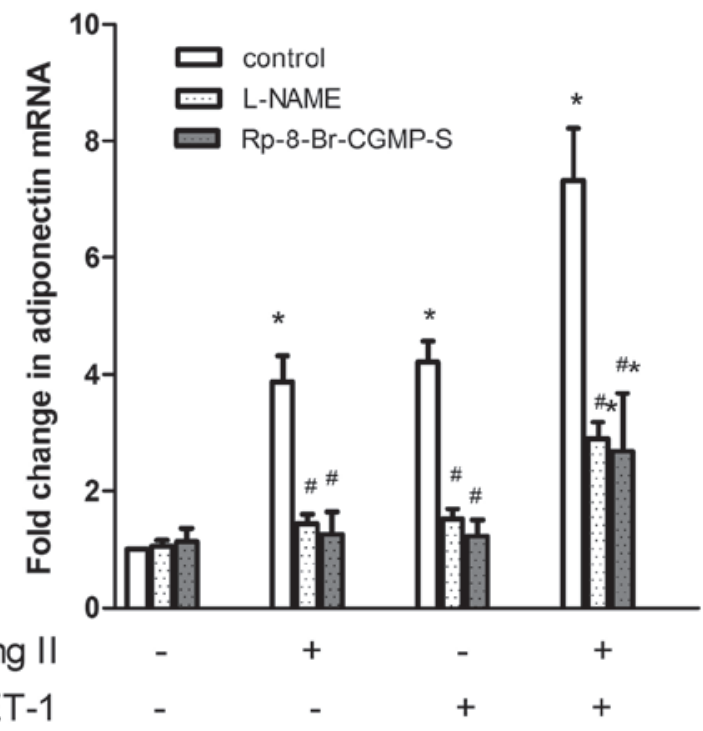

Figure 4. Effect of L-NAME and Rp-8-Br-cGMP-S on the mRNA expression of APN in response to ET-1, AngII and a combination of both. NRVMs were pre-incubated for $1 \mathrm{~h}$ in the presence or absence of L-NAME $\left(10^{-3} \mathrm{M}\right)$ or Rp-8-Br-cGMP-S $\left(10^{-5} \mathrm{M}\right)$ and were subsequently incubated in the presence or absence of ET-1, AngII and a combination of both for a further $24 \mathrm{~h}$ prior to the measurement of the mRNA expression of APN by reverse transcription-quantitative polymerase chain reaction. The data are presented as the mean \pm standard error of the mean $\left({ }^{*} \mathrm{P}<0.05\right.$ compared with the corresponding control, ${ }^{\#} \mathrm{P}<0.05$ compared with the corresponding none). ET-1, endothelin-1; AngII, angiotensin II; APN, adiponectin; NRVMs, neonatal rat ventricular myocytes.

through the incubation of the cells with the NOS inhibitor, L-NAME, and cGMP antagonist analogue, Rp-8-Br-cGMP-S.

Skurk et al (15) reported that APN is expressed in the hearts of patients with dilated cardiomyopathy (DCM), 
independent of their plasma levels; there is a local paracrine cardiac APN system involved in the pathogenesis of DCM. Cardiomyocyte-derived APN may have a paracrine effect on the cardiovascular system; APN serves a protective role in suppressing the development of cardiac hypertrophy and inhibits cardiovascular disease progression (16). It is very likely that upregulated ET and AngII in CHF induced cardiac APN expression, exerting its protective effects on the cardiovascular system and increasing the levels of APN in the peripheral circulation.

ET-1 and AngII serve important roles in the structural and electrical remodelling of the myocardium. They compose a mutually reciprocal signalling network in the myocardium and have certain reciprocal associations at the receptor level. AngII has been reported to upregulate the expression of $\mathrm{ET}_{\mathrm{B}} \mathrm{R}$ via the $\mathrm{AT}_{1} \mathrm{R}$ (17). Although our previous study (10) demonstrated that AngII upregulated the expression of APN in NRVMs via the $\mathrm{AT}_{2} \mathrm{R}$, the present study demonstrated that selective antagonists for $\mathrm{ET}_{\mathrm{A}} \mathrm{R}$ and $\mathrm{ET}_{\mathrm{B}} \mathrm{R}$, as well as $\mathrm{AT}_{2} \mathrm{R}$ diminished the additive roles of ET-1 and AngII on APN activation, indicating that at the receptor level, ET-1 and AngII have a mutually reciprocal association that indirectly activates the NO/cGMP/PKG signalling pathway.

In conclusion, the present study demonstrated that ET-1 upregulated the gene expression and secretion of APN in NRVMs, and that ET-1 and AngII serve additive roles in APN activation in NRVMs through the common NO/cGMP/PKG signalling pathway. Future studies will focus on the evaluation of how APN affects the heart and how APN can be used as a therapeutic treatment for heart disease.

\section{Acknowledgements}

This present study was supported by grants from the National Natural Science Foundation of China (nos. 81400217 and 81570345), the nature science foundation of Hebei Province (no. H2014206389) and Hebei Provincial Health Bureau of medical key subject (no. 20130156).

\section{References}

1. Correa MV, Nolly MB, Caldiz CI, de Cingolani GE, Cingolani HE and Ennis IL: Endogenous endothelin 1 mediates angiotensin II-induced hypertrophy in electrically paced cardiac myocytes through EGFR transactivation, reactive oxygen species and NHE-1. Pflugers Arch 466: 1819-1830, 2014.

2. de Jonge HW, Dekkers DH, Houtsmuller AB, Sharma HS and Lamers JM: Differential signaling and hypertrophic responses in cyclically stretched vs endothelin-1 stimulated neonatal rat cardiomyocytes. Cell Biochem Biophys 47: 21-32, 2007.
3. Lin YJ, Kwok CF, Juan CC, Hsu YP, Shih KC, Chen CC and Ho LT: Angiotensin II enhances endothelin-1-induced vasoconstriction through upregulating endothelin type A receptor. Biochem Biophys Res Commun 451: 263-269, 2014.

4. Wolf AM, Wolf D, Avila MA, Moschen AR, Berasain C, Enrich B Rumpold $\mathrm{H}$ and Tilg $\mathrm{H}$ : Up-regulation of the anti-inflammatory adipokine adiponectin in acute liver failure in mice. J Hepatol 44: 537-543, 2006.

5. Piñeiro R, Iglesias MJ, Gallego R, Raghay K, Eiras S, Rubio J, Diéguez C, Gualillo O, González-Juanatey JR and Lago F: Adiponectin is synthesized and secreted by human and murine cardiomyocytes. FEBS Lett 579: 5163-5169, 2005.

6. Kistorp C, Faber J, Galatius S, Gustafsson F, Frystyk J, Flyvbjerg A and Hildebrandt P: Plasma adiponectin, body mass index, and mortality in patients with chronic heart failure. Circulation 112: 1756-1762, 2005.

7. George J, Patal S, Wexler D, Sharabi Y, Peleg E, Kamari Y, Grossman E, Sheps D, Keren G and Roth A: Circulating adiponectin levels predict outcome in patients with severe congestive heart failure. Heart 92: 1420-1424, 2006.

8. Wannamethee SG, Whincup PH, Lennon L and Sattar N: Circulating adiponectin levels and mortality in elderly men with and without cardiovascular disease and heart failure. Arch Intern Med 167: 1510-1517, 2007.

9. Takano H, Obata JE, Kodama Y, Kitta Y, Nakamura T, Mende A, Kawabata K, Saito Y, Fujioka D, Kobayashi T, et al: Adiponectin is released from the heart in patients with heart failure. Int $\mathrm{J}$ Cardiol 132: 221-226, 2009.

10. Guo B, Li Y, Han R, Zhou H and Wang M: Angiotensin II upregulation of cardiomyocyte adiponectin production is nitric oxide/cyclic GMP dependent. Am J Med Sci 341: 350-355, 2011.

11. Livak KJ and Schmittgen TD: Analysis of relative gene expression data using real-time quantitative PCR and the 2(-Delta Delta C(T)) Method. Methods 25: 402-408, 2001.

12. Juan CC, Chuang TY, Chang CL, Huang SW and Ho LT: Endothelin-1 regulates adiponectin gene expression and secretion in 3T3-L1 adipocytes via distinct signaling pathways. Endocrinology 148: 1835-1842, 2007.

13. Bedi D, Clarke KJ, Dennis JC, Zhong Q, Brunson BL, Morrison EE and Judd RL: Endothelin-1 inhibits adiponectin secretion through a phosphatidylinositol 4,5-bisphosphate/actin-dependent mechanism. Biochem Biophys Res Commun 345: 332-339, 2006.

14. Yin WH, Chen YH, Wei J, Jen HL, Huang WP, Young MS, Chen DC and Liu PL: Associations between endothelin-1 and adiponectin in chronic heart failure. Cardiology 118: 207-216, 2011.

15. Skurk C, Wittchen F, Suckau L, Witt H, Noutsias M, Fechner H, Schultheiss HP and Poller W: Description of a local cardiac adiponectin system and its deregulation in dilated cardiomyopathy. Eur Heart J 29: 1168-1180, 2008.

16. Wang Y, Lau WB, Gao E, Tao L, Yuan Y, Li R, Wang X, Koch WJ and Ma XL: Cardiomyocyte-derived adiponectin is biologically active in protecting against myocardial ischemia-reperfusion injury. Am J Physiol Endocrinol Metab 298: E663-E670, 2010.

17. Kanno K, Hirata Y, Tsujino M, Imai T, Shichiri M, Ito H and Marumo F: Up-regulation of ETB receptor subtype mRNA by angiotensin II in rat cardiomyocytes. Biochem Biophys Res Commun 194: 1282-1287, 1993. 